\title{
CONVEX SETS WITH SEMIDEFINITE REPRESENTATION
}

\author{
JEAN B. LASSERRE
}

\begin{abstract}
We provide a sufficient condition on a class of compact basic semialgebraic sets $\mathbf{K} \subset \mathbb{R}^{n}$ for their convex hull $\operatorname{co}(\mathbf{K})$ to have a semidefinite representation (SDr). This SDr is explicitly expressed in terms of the polynomials $g_{j}$ that define $\mathbf{K}$. Examples are provided. We also provide an approximate SDr; that is, for every fixed $\epsilon>0$, there is a convex set $\mathbf{K}_{\epsilon}$ such that $\operatorname{co}(\mathbf{K}) \subseteq \mathbf{K}_{\epsilon} \subseteq \operatorname{co}(\mathbf{K})+\epsilon \mathbf{B}$ (where $\mathbf{B}$ is the unit ball of $\mathbb{R}^{n}$ ), and $\mathbf{K}_{\epsilon}$ has an explicit SDr in terms of the $g_{j}$ 's. For convex and compact basic semi-algebraic sets $\mathbf{K}$ defined by concave polynomials, we provide a simpler explicit SDr when the nonnegative Lagrangian $L_{f}$ associated with $\mathbf{K}$ and any linear $f \in \mathbb{R}[X]$ is a sum of squares. We also provide an approximate SDr specific to the convex case.
\end{abstract}

\section{INTRODUCTION}

An important issue raised in e.g. Ben-Tal and Nemirovski [2], Helton and Vinnikov [4], Parrilo and Sturmfels [14], is to characterize convex sets of $\mathbb{R}^{n}$ that have a lifted LMI (Linear Matrix Inequalities) (or a semidefinite representation $(\mathrm{SDr})$ ), and called SDr sets in [2] (that is, sets which are semidefinite representable).

Recall that a convex set $\Omega \subset \mathbb{R}^{n}$ is SDr if there exist integers $m, p$ and real $p \times p$ symmetric matrices $\left\{A_{i}\right\}_{i=0}^{n},\left\{B_{j}\right\}_{j=1}^{m}$ such that:

$$
\Omega=\left\{x \in \mathbb{R}^{n}: \exists y \in \mathbb{R}^{m} \text { s.t. } A_{0}+\sum_{i=1}^{n} A_{i} x_{i}+\sum_{j=1}^{m} B_{j} y_{j} \succeq 0\right\}
$$

(where the notation $A \succeq 0$ stands for the matrix $A$ is positive semidefinite). In other words, $\Omega$ is the linear projection on $\mathbb{R}^{n}$ of the convex set

$$
\Omega^{\prime}:=\left\{(x, y) \in \mathbb{R}^{n} \times \mathbb{R}^{m}: A_{0}+\sum_{i=1}^{n} A_{i} x_{i}+\sum_{j=1}^{m} B_{j} y_{j} \succeq 0\right\}\left(\subset \mathbb{R}^{n+m}\right)
$$

of the lifted space $\mathbb{R}^{n+m}$. The set $\Omega^{\prime}$ is called a semidefinite representation (SDr) of $\Omega$ and is a lifted LMI because one sometimes needs additional variables $y \in \mathbb{R}^{m}$ to obtain a description of $\Omega$ via appropriate LMIs. For instance:

1991 Mathematics Subject Classification. 52A20 52A27 52A41 90C22 90C25 13B25 $12 \mathrm{D} 15$.

Key words and phrases. Convex sets; semidefinite representation; representation of positive polynomials; sum of squares. 
- The intersection of half-spaces, i.e., a polyhedron $\left\{x \in \mathbb{R}^{n}: A x \leq b\right\}$, is a trivial example of convex sets whose SDr is readily available without lifting. Indeed $A x \leq b$ is an LMI with diagonal matrices $A_{i}$ in (1.1).

- The intersection of ellipsoids

$$
\Omega:=\left\{x \in \mathbb{R}^{n}: x^{T} Q_{j} x+b^{T} x+c_{j} \geq 0, j=1, \ldots, m\right\}
$$

(where $-Q_{j} \succeq 0$ for all $j=, \ldots, m$ ) is a SDr set with lifted LMI representation in $\mathbb{R}^{(n+1)(n+2) / 2-1}$ :

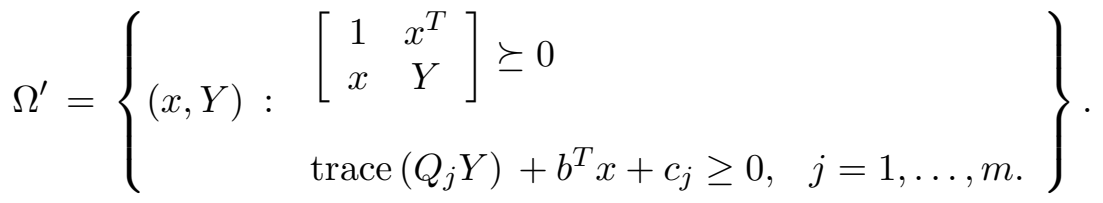

- The epigraph of a univariate convex polynomial is a SDr set.

- Convex sets of $\mathbb{R}^{2}$ described from genus-zero plane curves are SDr sets; see Parrilo [14].

- Hyperbolic cones obtained from 3-variables hyperbolic homogeneous polynomials are SDr sets; see the proof of the Lax conjecture in Lewis et al. [13].

So far, and except for the special cases cited above, little is known. In addition, even if a convex set $\mathbf{K}$ is known to be SDr, there is no systematic procedure to obtain its SDr, i.e., the set of lifted LMIs whose projection describe K. However, Helton and Vinnikov [4] have proved recently that rigid convexity is a necessary condition for a set to be SDr (and sufficient for dimension $n=2$ ). Chua and Tuncel [3] consider even more general lifted conic representations of convex sets, called lifted G-representations (SDr being a special case) and discuss various geometric properties of convex sets admitting such lifted G-representations, as well as measures of "goodness" for such representations.

In this paper, we consider the convex hull $\operatorname{co}(\mathbf{K})$ of compact basic semialgebraic sets $\mathbf{K} \subset \mathbb{R}^{n}$ of the form

$$
\mathbf{K}=\left\{x \in \mathbb{R}^{n}: \quad g_{j}(x) \geq 0, \quad j=1, \ldots, m\right\},
$$

for some given polynomials $g_{j} \in \mathbb{R}[X], j=1, \ldots, m$. Notice that the class of sets (1.3) is fairly general as $\mathbf{K}$ can be nonconvex (even disconnected), as well as discrete.

Contribution: Our contribution is twofold:

I. We first provide a sufficient condition (and a variant of it) on the defining polynomials $\left(g_{j}\right) \subset \mathbb{R}[X]$ of $\mathbf{K}$ that we call Schmüdgen's Bounded Degree Representation (S-BDR) of affine polynomials and its Putinar-Prestel variant (PP-BDR). A basic compact semi-algebraic set has the S-BDR (resp. 
PP-BDR) property if almost all affine polynomials $f \in \mathbb{R}[X]$ positive on $\mathbf{K}$ (hence on $\operatorname{co}(\mathbf{K})$ ) belong to $P_{r}(g)$ (resp. $Q_{r}(g)$ ), a subset of the preordering $P(g)$ (resp. quadratic module $Q(g))$ generated by the $g_{j}$. When $f \in P_{r}(g)$ or $Q_{r}(g)$, all elements in the representation of $f$ in the preordering $P(g)$ or in the quadratic module $Q(g)$, have degree at most $r$.

Recall that when $\mathbf{K}$ is compact then $f>0$ on $\mathbf{K}$ implies $f \in P(g)$ (or $f \in Q(g)$ if $N-\|X\|^{2} \in Q(g)$ for some $N$ ), and so the S-BDR (or PP-BDR) property is stronger in that it requires $f \in P_{r}(g)$ (or $Q_{r}(g)$ ). On the other hand, this requirement is only concerned with the class of positive affine polynomials.

For instance, this property holds for intersections of halfspaces and ellipsoids, i.e., when the $g_{j}$ 's are affine or quadratic and concave. But we also exhibit some nontrivial non convex compact semi-algebraic sets $\mathbf{K}$ with the PP-BDR property. For instance, we show that when $m=2$ and the $g_{j}$ 's are quadratic, or when $n=2$ and the $g_{j}$ 's are quartic, then the PP-BDR property holds generically, and with order $r=1$ and $r=2$, respectively.

When the S-BDR or PP-BDR property holds then one can immediately obtain an explicit SDr of co(K), expressed directly in terms of the defining polynomials $g_{j}$.

We also obtain an approximate result of the following flavor. For every fixed $\epsilon>0$, we exhibit a convex set $\mathbf{K}_{\epsilon}$ such that (a) $\operatorname{co}(\mathbf{K}) \subseteq \mathbf{K}_{\epsilon} \subseteq$ $\operatorname{co}(\mathbf{K})+\epsilon \mathbf{B}$ (where $\mathbf{B}$ is the unit ball of $\mathbb{R}^{n}$ ), and (b) $\mathbf{K}_{\epsilon}$ has an explicit $\mathrm{SDr}$ expressed directly in terms of the polynomials $g_{j}$ that define $\mathbf{K}$. This result improves significantly upon [8] where we have provided outer convex approximations of $\operatorname{co}(\mathbf{K})$, i.e., a monotone nonincreasing sequence of convex sets $\mathbf{K}_{r}$, with $\mathbf{K}_{r} \downarrow \operatorname{co}(\mathbf{K})$, and where each $\mathbf{K}_{r}$ has a SDr. And so, if $x \notin \operatorname{co}(\mathbf{K})$ then $x \notin \mathbf{K}_{r}$ for all $r \geq r(x)$ for some $r(x)$ that depends on $x$, an undesirable feature.

II. However, for general basic semialgebraic sets $\mathbf{K}$, one cannot expect that the S-BDR (or PP-BDR) property holds (if it ever holds) for nice values of the order $r$. Indeed otherwise one could minimize any affine polynomial on $\mathbf{K}$ efficiently. Therefore, from a practical point of view, the most interesting case is essentially when $\mathbf{K}$ is convex ... and even more ... when the defining polynomials $g_{j}$ in (1.3) are concave, because then one may hope for the S-BDR or PP-BDR property to hold for interesting values of $r$.

So, our second contribution is concerned with the case of compact convex basic semialgebraic sets $\mathbf{K}$ defined by concave polynomials. We first show that the PP-BDR property holds for $\mathbf{K}$ whenever the Lagrangian $L_{f}$ associated with $\mathbf{K}$ and an arbitrary linear $f \in \mathbb{R}[X]$ is a sum of squares (s.o.s.) (by construction it is already nonnegative). In this case, $\mathbf{K}$ has a natural SDr based on the Karush-Kuhn-Tucker optimality conditions. This makes an interesting connection between convexity and s.o.s. Finally, we also provide an approximate $\mathrm{SDr}$ of $\mathbf{K}$, specific to the convex case. 


\section{I. Semidefinite Representation of $\operatorname{co}(\mathbf{K})$}

2.1. Notation and definitions. For a real symmetric matrix $A$ the notation $A \succeq 0$ (resp. $A \succ 0$ ) stands for $A$ is positive semidefinite (resp. positive definite). Let $\mathbb{R}[X]$ be the ring of real polynomials in the variables $X=\left(X_{1}, \ldots, X_{n}\right)$ and let $\Sigma^{2} \subset \mathbb{R}[X]$ be its subset of sums of squares (s.o.s.) (whereas $\Sigma_{d}^{2}$ is that of degree at most $2 d$ ). For $x \in \mathbb{R}^{n}$, let $\|x\|$ denote its euclidean norm.

With $d \in \mathbb{N}$, let $s(d):=\left(\begin{array}{c}n+d \\ n\end{array}\right)$, and let $u(X) \in \mathbb{R}^{s(d)}$ be the column vector

$$
u_{d}(X)=\left(1, X_{1}, \ldots, X_{n}, X_{1}^{2}, X_{1} X_{2}, \ldots, X_{n}^{d}\right)^{T},
$$

whose components form the usual canonical basis of the vector space $\mathbb{R}[X]_{d}$ (of dimension $s(d)$ ) of real polynomials of degree at most $d$.

Given a infinite sequence $y:=\left\{y_{\alpha}\right\}_{\alpha \in \mathbb{N}^{n}}$ indexed in the canonical basis $u_{\infty}(X)$, let $L_{y}: \mathbb{R}[X] \rightarrow \mathbb{R}$ be the linear mapping

$$
f \in \mathbb{R}[X]\left(=\sum_{\alpha \in \mathbb{N}^{n}} f_{\alpha} X^{\alpha}\right) \longmapsto L_{y}(f):=\sum_{\alpha \in \mathbb{N}^{n}} f_{\alpha} y_{\alpha},
$$

and let $\mathbf{f}=\left\{f_{\alpha}\right\} \in \mathbb{R}^{s(d)}$ be the vector of coefficients of $f \in \mathbb{R}[X]_{d}$ in the basis $u_{d}(X)$.

Moment matrix. Let $M_{d}(y)$ be the $s(d) \times s(d)$ real matrix with rows and columns indexed in the basis $u_{d}(X)$, and defined by:

$$
M_{d}(y)(\alpha, \beta)=y_{\alpha+\beta}, \quad \alpha, \beta \in \mathbb{N}^{n}, \quad|\alpha|,|\beta| \leq d,
$$

where for every $\alpha \in \mathbb{N}^{n}$, the notation $|\alpha|$ stands for $\sum_{i=1}^{n} \alpha_{i}$.

Equivalently, $M_{d}(y)=L_{y}\left(u_{d}(X) u_{d}(X)^{T}\right)$, meaning that $L_{y}$ is applied entrywise to the polynomial matrix $u_{d}(X) u_{d}(X)^{T}$. The matrix $M_{d}(y)$ is called the moment matrix associated with the sequence $y$; see e.g. [9]. If $y$ has a representing measure $\mu_{y}$ (i.e., if $y_{\alpha}=\int X^{\alpha} d \mu_{y}$ for every $\alpha \in \mathbb{N}^{n}$ ) then, one has

$$
\left\langle\mathbf{f}, M_{d}(y) \mathbf{f}\right\rangle=\int f^{2} d \mu_{y} \geq 0, \quad \forall f \in \mathbb{R}[X]_{d},
$$

so that $M_{d}(y) \succeq 0$.

Localizing matrix. Similarly, given $y=\left\{y_{\alpha}\right\}$ and $\theta \in \mathbb{R}[X]$, let $M_{d}(\theta y)$ be the $s(d) \times s(d)$ matrix defined by:

$$
M_{d}(\theta y):=L_{y}\left(\theta(X) u_{d}(X) u_{d}(X)^{T}\right)
$$

i.e., $L_{y}$ is applied entrywise to the polynomial matrix $\theta(X) u_{d}(X) u_{d}(X)^{T}$. The matrix $M_{d}(\theta y)$ is called the localizing matrix associated with the sequence $y$ and the polynomial $\theta$ (see again [9]). Notice that the localizing matrix with respect to the constant polynomial $\theta \equiv 1$ is the moment matrix $M_{d}(y)$ in $(2.2)$. 
If $y$ has a representing measure $\mu_{y}$ with support contained in the level set $\left\{x \in \mathbb{R}^{n}: \theta(x) \geq 0\right\}$ (where $\theta \in \mathbb{R}[X]$ ), then

$$
\left\langle\mathbf{f}, M_{d}(\theta y) \mathbf{f}\right\rangle=\int f^{2} \theta d \mu_{y} \geq 0 \quad \forall f \in \mathbb{R}[X]_{d},
$$

so that $M_{d}(\theta y) \succeq 0$.

2.2. Semidefinite representation of $\operatorname{co}(\mathbf{K})$. Let $\mathbf{K} \subset \mathbb{R}^{n}$ be the basic closed semi-algebraic set defined in (1.3) for some polynomials $g_{j} \in \mathbb{R}[X]$, $j=1, \ldots, m$.

For every $J \subseteq\{1, \ldots, m\}$, let $g_{J}:=\prod_{j \in J} g_{j}$, with the convention $g_{\emptyset} \equiv 1$, and let $P(g) \subset \mathbb{R}[X]$ be the preordering generated by the $g_{j}$ 's, i.e.,

$$
P(g):=\left\{\sum_{J \subseteq\{1, \ldots, m\}} \sigma_{J} g_{J}: \sigma_{J} \in \Sigma^{2}\right\},
$$

and given $r \in \mathbb{N}$, define $P_{r}(g) \subset P(g)$ to be the set

$$
P_{r}(g):=\left\{\sum_{J \subseteq\{1, \ldots, m\}} \sigma_{J} g_{J}: \quad \sigma_{J} \in \Sigma^{2}, \operatorname{deg} \sigma_{J}+\operatorname{deg} g_{J} \leq 2 r\right\} .
$$

Similarly, let $Q(g) \subset \mathbb{R}[X]$ be the quadratic module generated by the $g_{j}$ 's, i.e.,

$$
Q(g):=\left\{\sum_{j=0}^{m} \sigma_{j} g_{j}: \quad \sigma_{j} \in \Sigma^{2}\right\}
$$

(with the convention $g_{0} \equiv 1$ ), and given $r \in \mathbb{N}$, define $Q_{r}(g) \subset Q(g)$ to be the set

$$
Q_{r}(g):=\left\{\sum_{j=0}^{m} \sigma_{j} g_{j}: \quad \sigma_{j} \in \Sigma^{2}, \operatorname{deg} \sigma_{j}+\operatorname{deg} g_{j} \leq 2 r\right\} .
$$

Definition 1 (Semi Definite representation (SDr)). A convex set $\Omega \subset \mathbb{R}^{n}$ has a $\mathrm{SDr}$ (or is a SDr set) if it has the form

$$
\boldsymbol{\Omega}=\left\{x \in \mathbb{R}^{n}: \quad \exists y \in \mathbb{R}^{m} \text { s.t. } \quad A_{0}+\sum_{i=1}^{n} A_{i} x_{i}+\sum_{k=1}^{p} B_{k} y_{k} \succeq 0\right\}
$$

for some integer $p$ and real symmetric matrices $\left\{A_{i}\right\}$ and $\left\{B_{k}\right\}$.

For an affine polynomial $X \mapsto f_{0}+\sum_{i=1}^{n} f_{i} X_{i}$, let $\left(f_{0}, \mathbf{f}\right) \in \mathbb{R} \times \mathbb{R}^{n}$ be its vector of coefficients.

Definition 2 (Schmüdgen's Bounded Degree Representation of affine polynomials). Given a compact set $\mathbf{K} \subset \mathbb{R}^{n}$ defined as in (1.3), one says that Schmüdgen's Bounded Degree Representation (S-BDR) of affine polynomials holds for $\mathbf{K}$ if there exists $r \in \mathbb{N}$ such that

$$
[f \text { affine and positive on } \mathbf{K}] \Rightarrow f \in P_{r}(g) \text {, }
$$


except perhaps on a set of vectors $\mathbf{f}$ in $\mathbb{R}^{n}$ with Lebesgue measure zero. Call $r$ its order.

Definition 3 (Putinar-Prestel's Bounded Degree Representation of affine polynomials). Given a compact set $\mathbf{K} \subset \mathbb{R}^{n}$ defined as in (1.3), one says that Putinar-Prestel's Bounded Degree Representation (PP-BDR) of affine polynomials holds for $\mathbf{K}$ if there exists $r \in \mathbb{N}$ such that

$$
\text { [ } f \text { affine and positive on } \mathbf{K}] \Rightarrow f \in Q_{r}(g) \text {, }
$$

except perhaps on a set of vectors $\mathbf{f}$ in $\mathbb{R}^{n}$ with Lebesgue measure zero. Call $r$ its order.

Remark 1. (a) Observe that if $\mathbf{K}$ is compact, by Schmüdgen's Positivstellensatz [17]

$$
[f \in \mathbb{R}[X] \text { and } f \text { positive on } \mathbf{K}] \Rightarrow f \in P_{r}(g),
$$

for some $r(f)$. The S-BDR property states that $r(f)<r$ for almost all affine $f \in \mathbb{R}[X]$, positive on $\mathbf{K}$.

(b) If for some $N$, the polynomial $N-\|X\|^{2}$ is in $Q(g)$, then by Putinar's Positivstellensatz [16]

$$
[f \in \mathbb{R}[X] \text { and } f \text { positive on } \mathbf{K}] \Rightarrow f \in Q_{r}(g),
$$

for some $r(f)$; see also Jacobi and Prestel [7]. The PP-BDR property states that $r(f)<r$ for almost all affine $f \in \mathbb{R}[X]$, positive on $\mathbf{K}$.

(c) Finally, the PP-BDR property implies the S-BDR property.

For every $J \subseteq\{1, \ldots, m\}$ let $r_{J}:=\left\lceil\operatorname{deg} g_{J} / 2\right\rceil$.

Theorem 2. Let $\mathbf{K} \subset \mathbb{R}^{n}$ be compact and defined as in (1.3).

(a) If the $S$-BDR property holds for $\mathbf{K}$ with order $r$, then $\operatorname{co}(\mathbf{K})$ is a $S D r$ set with $S D r$

$$
\left\{(x, y) \in \mathbb{R}^{n} \times \mathbb{R}^{s(2 r)}:\left\{\begin{array}{lll}
M_{r-r_{J}}\left(g_{J} y\right) & \succeq 0, \quad J \subseteq\{1, \ldots, m\} \\
L_{y}\left(X_{i}\right) & =x_{i}, \quad i=1, \ldots, n \\
y_{0} & =1
\end{array}\right\} .\right.
$$

(b) If the PP-BDR property holds for $\mathbf{K}$ with order $r$, then $\operatorname{co}(\mathbf{K})$ is a SDr set with $S D r$

$$
\left\{(x, y) \in \mathbb{R}^{n} \times \mathbb{R}^{s(2 r)}:\left\{\begin{array}{lll}
M_{r-r_{j}}\left(g_{j} y\right) & \succeq 0, \quad j=0,1, \ldots, m \\
L_{y}\left(X_{i}\right) & =x_{i}, & i=1, \ldots, n \\
y_{0} & =1
\end{array}\right\} .\right.
$$

Proof. We only prove (a) as (b) is proved in exactly the same manner. Let $\Omega \subset \mathbb{R}^{n} \times \mathbb{R}^{s(r)}$ be the set defined in (2.11). We have to show that ( $\exists y$ : $(x, y) \in \Omega) \Leftrightarrow x \in \operatorname{co}(\mathbf{K})$. 
1. $x \in \operatorname{co}(\mathbf{K}) \Rightarrow(x, y) \in \Omega$ for some $y \in \mathbb{R}^{s(r)}$. Observe that by the definition of $\operatorname{co}(\mathbf{K})$,

$$
x \in \operatorname{co}(\mathbf{K}) \quad \Leftrightarrow \quad x=\int X d \mu
$$

for some probability measure $\mu$ supported on $\mathbf{K}$. Let $y=\left(y_{\alpha}\right) \in \mathbb{R}^{s(r)}$ be the sequence of moments of $\mu$ up to order $2 r$, i.e.

$$
y_{\alpha}=L_{y}\left(X^{\alpha}\right)=\int X^{\alpha} d \mu, \quad \alpha \in \mathbb{N}^{n} ;|\alpha| \leq 2 r .
$$

The sequence $y$ is well defined because $\mu$ has compact support; in particular $y_{0}=1$. From the definition of $\mu$ one has $L_{y}\left(X_{i}\right)=\int X_{i} d \mu=x_{i}$. In addition, as $\mu$ is supported on $\mathbf{K}$, one has $M_{r-r_{J}}\left(g_{J} y\right) \succeq 0$ for all subsets $J \subseteq\{1, \ldots, m\}$ (just take $\theta:=g_{J}$ in $(2.5)$ ). And so $(x, y) \in \Omega$.

2. $\exists y:(x, y) \in \Omega \Rightarrow x \in \operatorname{co}(\mathbf{K})$. We prove it by contradiction.

Let $x \notin \mathrm{co}(\mathbf{K})$ and assume that there exists $y \in \mathbb{R}^{s(r)}$ such that $(x, y) \in \Omega$. As $\operatorname{co}(\mathbf{K})$ is convex and compact, by the Hahn-Banach separation theorem, there exists $\left(f_{0}, \mathbf{f}\right) \in \mathbb{R} \times \mathbb{R}^{n}$ such that

$$
\langle\mathbf{f}, x\rangle<f_{0} \quad \text { and } \quad\langle\mathbf{f}, z\rangle>f_{0} \quad \forall z \in \operatorname{co}(\mathbf{K}),
$$

and so the affine polynomial $f \in \mathbb{R}[X], X \mapsto f(X):=-f_{0}+\sum_{i} f_{i} X_{i}$ is positive on $\mathbf{K}$.

By the PP-BDR property of $\mathbf{K}$ with order $r$, one has $f \in P_{r}(g)$ or $\tilde{f} \in$ $P_{r}(g)$ for some affine $\tilde{f} \in \mathbb{R}[X]$ with coefficient vector $\left(\tilde{\mathbf{f}}, \tilde{f}_{0}\right)$ such that $\|\tilde{\mathbf{f}}\|=1$ and some $\epsilon>0$ such that $\left\|\left(\tilde{\mathbf{f}}, \tilde{f}_{0}\right)-\left(\mathbf{f}, f_{0}\right)\right\|<\epsilon$, with $\epsilon>0$ as small as desired. Therefore, one may choose $\epsilon$ sufficiently small to ensure that $\left(\tilde{\mathbf{f}}, \tilde{f}_{0}\right)$ also satisfies $(2.13)$ and so, one may rename $\tilde{f}$ as $f$ and safely assume that $f \in P_{r}(g)$. Hence,

$$
f(X)=\sum_{J \subseteq\{1, \ldots, m\}} \sigma_{J} g_{J}, \quad \sigma_{J} \in \Sigma^{2} ; \operatorname{deg} \sigma_{J}+\operatorname{deg} g_{J} \leq 2 r .
$$

Observe that as $\sigma_{J}$ is s.o.s. and $\operatorname{deg} \sigma_{J}+\operatorname{deg} g_{J} \leq 2 r$, one has

$$
L_{y}\left(\sigma_{J} g_{J}\right) \geq 0 \quad \forall J \subseteq\{1, \ldots, m\} .
$$

Applying the linear functional $L_{y}$ to the polynomial $f$ in (2.14) yields the contradiction

$$
\begin{aligned}
0>\mathbf{f}^{T} x-f_{0} & =-f_{0} y_{0}+\sum_{i=1}^{n} L_{y}\left(f_{i} X_{i}\right) \quad\left[\text { as } y_{0}=1, L_{y}\left(X_{i}\right)=x_{i} \forall i\right] \\
& =L_{y}(f(X))=\sum_{J \subseteq\{1, \ldots, m\}} L_{y}\left(g_{J} \sigma_{J}\right) \quad[\text { by }(2.14)] \\
& \geq 0 \quad[\text { by }(2.15)] .
\end{aligned}
$$

This proves that there is no $y$ such that $(x, y) \in \Omega$, the desired result.

Notice that in Theorem 2, the SDr (2.11) and (2.12) of $\operatorname{co}(\mathbf{K})$ are given explicitly in terms of the data $g_{j}$ 's that define $\mathbf{K}$. 
2.3. Examples of convex K. We have already seen that the intersection $\mathbf{K}$ of half-spaces and/or ellipsoids is a SDr set. But we here show that the PP-BDR property holds for such sets $\mathbf{K}$, and also for the intersection of level sets of quartic polynomials in two variables. Of course, one already knows how to build up a SDr for $\mathbf{K}$ at least in the first two cases. But this is to illustrate that the domain of application of Theorem 2 is not empty and not trivial.

Example 1. Let us start with $\mathbf{K}$ being a convex polytope defined by linear inequalities, i.e., $g_{j} \in \mathbb{R}[X]$ is affine in $X$ for all $j=1, \ldots, m$. Hence $\operatorname{co}(\mathbf{K}) \equiv \mathbf{K}$ and this description of $\mathbf{K}$ by the $g_{j}$ 's is already a SDr; it is even a linear system. We briefly prove that the PP-BDR property holds for $\mathbf{K}$ with order 0 . Let $f \in \mathbb{R}[X]$ be affine with coefficient vector $\left(f_{0}, \mathbf{f}\right) \in \mathbb{R} \times \mathbb{R}^{n}$, and write

$$
\begin{aligned}
g_{j}(X) & =g_{j 0}+\sum_{i=1}^{n} g_{j i} X_{i}, \quad j=1, \ldots, m \\
f(X) & =f_{0}+\sum_{i=1}^{n} f_{i} X_{i} .
\end{aligned}
$$

Next, let $G \in \mathbb{R}^{m \times n}$ be the matrix $G(j, i)=g_{j i}, j=1, \ldots, m, i=1, \ldots, n$, and $\mathbf{g}=\left(g_{j 0}\right) \in \mathbb{R}^{m}$. If $f$ is nonnegative on $\mathbf{K}$ then by Farkas lemma $\mathbf{f}=\lambda^{T} G$ and $f_{0} \geq \lambda^{T} \mathbf{g}$, for some nonnegative vector $\lambda \in \mathbb{R}^{m}$. Therefore $f(X)=u+\sum_{j=1}^{m} \lambda_{j} g_{j}(X)$, for some nonnegative scalar $u$, which proves that $f \in Q_{1}(g)$. That is, the PP-BDR property holds for $\mathbf{K}$ with order $r=1$.

Example 2. Let $g_{j} \in \mathbb{R}[X]$ be concave and quadratic, for all $j=1, \ldots, m$. Then $\mathbf{K}$ is convex and it is well-known that $\mathbf{K}$ is a SDr set. Let $f \in \mathbb{R}[X]$ be affine with coefficient vector $\left(f_{0}, \mathbf{f}\right) \in \mathbb{R} \times \mathbb{R}^{n}$, and nonnegative on $\mathbf{K}$, so that $f^{*}:=\min _{x \in \mathbf{K}} f(x) \geq 0$. Assume that $\mathbf{K}$ is compact with nonempty interior. Convexity along with Slater's condition ${ }^{1}$ imply that the KKT optimality conditions hold at any global minimizer $x^{*} \in \mathbf{K}$, i.e.,

$$
\mathbf{f}-\sum_{j=1}^{m} \lambda_{j} \nabla g_{j}\left(x^{*}\right)=0 ; \quad \lambda_{j} g_{j}\left(x^{*}\right)=0, j=1, \ldots, m,
$$

for some nonnegative Lagrange multipliers $\lambda \in \mathbb{R}_{+}^{m}$. Then $x^{*}$ is also a global minimizer of the (convex) quadratic Lagrangian $L_{f}:=f-\sum_{j=1}^{m} \lambda_{j} g_{j}$ on the whole $\mathbb{R}^{n}$. Therefore, $L_{f}-f^{*} \geq 0$ on $\mathbb{R}^{n}$ and being quadratic, $L_{f}-f^{*} \in \Sigma^{2}$. Hence

$$
f=f^{*}+\left(L_{f}-f^{*}\right)+\sum_{j=1} \lambda_{j} g_{j}
$$

\footnotetext{
${ }^{1}$ Slater's condition states that there exists $x_{0} \in \mathbf{K}$ such that $g_{j}\left(x_{0}\right)>0$ for every $j=1, \ldots, m$. If Slater's condition holds and $f$ is convex and differentiable, then the Karush-Kuhn-Tucker (KKT) optimality conditions hold at any minimizer $x^{*} \in \mathbf{K}$ of the convex optimization problem: $\min _{x}\{f(x): x \in \mathbf{K}\}$
} 
that is, $f \in Q_{1}(g)$ (as $f^{*} \geq 0$ ). And so the PP-BDR property holds for $\mathbf{K}$ with order $r=1$, and $\mathbf{K}$ has the $\operatorname{SDr}(2.12)$. Writing $g_{j}(x)=x^{T} Q_{j} x+$ $b^{T} x+c_{j}$ for some positive semidefinite matrix $-Q_{j} \succeq 0, j=1, \ldots, m$, the $\mathrm{SDr}(2.12)$ is nothing less than (1.2) already encountered in the introduction.

Example 3. Let $n=2$ with $g_{j}$ concave and $\operatorname{deg} g_{j}=2$ or 4 , for all $j=$ $1, \ldots, m$, so that $\mathbf{K}$ is convex. Assume $\mathbf{K}$ is compact with nonempty interior. It is known that in general $\mathbf{K}$ is not representable by a LMI in the variables $x_{1}$ and $x_{2}$ only;. For instance take $m=1$ and $g_{1}(X)=1-X_{1}^{4}-X_{2}^{4}$. The rigid convexity condition of Helton and Vinnikov [4] is violated, but on the other hand, $\mathbf{K}$ is known to be $\mathrm{SDr}$.

Let $f \in \mathbb{R}[X]$ be affine and nonnegative on $\mathbf{K}$ with global minimum $f^{*} \geq 0$ on K. Again, convexity along with Slater's condition implies that the KKT optimality conditions hold at any global minimizer $x^{*} \in \mathbf{K}$. And so there exist nonnegative Lagrange multipliers $\lambda \in \mathbb{R}_{+}^{m}$ such that the (convex) Lagrangian $L_{f}:=f-\sum_{j=1}^{m} \lambda_{j} g_{j}$ also has optimal value $f^{*}$ and, in addition, $x^{*} \in \mathbf{K}$ is a global minimizer of $L_{f}$ on $\mathbb{R}^{2}$. Therefore, the polynomial $L_{f}-f^{*}$ being nonnegative on $\mathbb{R}^{2}$ and being quadratic or quartic in 2 variables, is s.o.s. That is $L_{f}-f^{*}=\sigma$ for some $\sigma \in \Sigma^{2}$ and $\operatorname{deg} \sigma \leq 4$. But then

$$
f=f^{*}+\left(L_{f}-f^{*}\right)+\sum_{j=1}^{m} \lambda_{j} g_{j} \in Q_{2}(g)
$$

because as $f^{*} \geq 0, f^{*}+\left(L_{f}-f^{*}\right) \in \Sigma^{2}$. That is, the PP-BDR property holds for $\mathbf{K}$ with order $r=2$. Hence, $\mathbf{K}$ has the SDr (2.12).

\subsection{Examples with nonconvex $\mathbf{K}$.}

Example 4. Let $m=2$ with

$$
g_{i}(X)=X^{T} A_{i} X+c_{i}, \quad i=1,2,
$$

for some real symmetric matrices $A_{i}$, and vector $\mathbf{c}=\left(c_{1}, c_{2}\right) \in \mathbb{R}^{2}$.

Given a linear polynomial $f \in \mathbb{R}[X]$ with coefficient vector $\mathbf{f}=\left(f_{i}\right)_{i=1}^{n} \in$ $\mathbb{R}^{n}$, consider the SDP

$$
\text { Q: } \quad \min _{y}\left\{L_{y}(f): \quad M_{1}(y) \succeq 0 ; L_{y}\left(g_{i}\right) \geq 0 ; i=1,2 ; y_{0}=1\right\}
$$

with optimal value denoted $\inf \mathbf{Q}(\min \mathbf{Q}$ if the infimum is achieved at some $\left.y^{*}\right)$, and with dual

$$
\mathbf{Q}^{*}: \max _{\lambda, \gamma, \sigma}\left\{\gamma: f-\gamma=\sigma+\lambda_{1} g_{1}+\lambda_{2} g_{2} ; \lambda_{1}, \lambda_{2} \geq 0 ; \sigma \in \Sigma_{2}^{2}\right\}
$$

where $\Sigma_{2}^{2}$ is the set of s.o.s. of degree 2. Let $A_{\lambda}:=\lambda_{1} A_{1}+\lambda_{2} A_{2}$ and introduce the matrix

$$
H(\lambda, \gamma):=\left[\begin{array}{c|c}
-\gamma-\langle\lambda, \mathbf{c}\rangle & \mathbf{f}^{T} / 2 \\
------- & ------ \\
\mathbf{f} / 2 & -A_{\lambda}
\end{array}\right] .
$$


Then $\mathbf{Q}^{*}$ has the equivalent form

$$
\mathbf{Q}^{*}: \max _{\lambda \geq 0, \gamma}\{\gamma: \quad H(\lambda, \gamma) \succeq 0\}
$$

with optimal value denoted $\sup \mathbf{Q}^{*}\left(\max \mathbf{Q}^{*}\right.$ if the $\sup$ is achieved). Obviously

$$
\inf \mathbf{Q} \leq f^{*}:=\min _{x}\{f(x): \quad x \in \mathbf{K}\},
$$

and $\min \mathbf{Q}=f^{*}$ holds if for instance $M_{1}\left(y^{*}\right)$ is rank one at some optimal solution $y^{*}$. Indeed, in this case, $y^{*}=\left(1, x^{*},\left(x_{1}^{*}\right)^{2}, x_{1}^{*} x_{2}^{*}, \ldots,\left(x_{n}^{*}\right)^{2}\right)$, which implies $L_{y^{*}}(f)=f\left(x^{*}\right)$ and $L_{y^{*}}\left(g_{i}\right)=g_{i}\left(x^{*}\right) \geq 0, i=1,2$.

Theorem 3. Let $\mathbf{K} \subset \mathbb{R}^{n}$ be defined as in (1.3) with $m=2$ and $g_{j}$ as in (2.16), and let $\mathbf{Q}$ be as in (2.17). Assume that $\mathbf{K}$ is compact with nonempty interior and

$$
\lambda_{1} A_{1}+\lambda_{2} A_{2} \prec 0
$$

for some $\lambda=\left(\lambda_{1}, \lambda_{2}\right) \geq 0$. Then for generic $\mathbf{f} \in \mathbb{R}^{n}$ :

(a) $\min \mathbf{Q}=f^{*}$

(b) The PP-BDR property holds for $\operatorname{co}(\mathbf{K})$ with order $r=1$, and so $\operatorname{co}(\mathbf{K})$ has the $S D r$ (2.12), i.e.,

$$
\left\{M_{1}(y) \succeq 0 ; L_{y}\left(g_{j}\right) \geq 0, j=1,2 ; L_{y}\left(X_{i}\right)=x_{i}, i=1, \ldots, n ; y_{0}=1\right\} .
$$

Proof. (a) Slater's condition holds for $\mathbf{Q}$ and $\mathbf{Q}^{*}$. Indeed as $\mathbf{K}$ has nonempty interior, let $\mu$ be the uniform probability measure on $\mathbf{K}$, with (well-defined) sequence of moment $y=\left(y_{\alpha}\right)$ (hence with $\left.y_{0}=1\right)$. It satisfies $M_{1}(y) \succ 0$ and $L_{y}\left(g_{1}\right)>0$ as well as $L_{y}\left(g_{2}\right)>0$.

Next, in view of (2.20), one may find $\lambda_{1}, \lambda_{2}>0$ and $\gamma \in \mathbb{R}$ such that $A_{\lambda} \prec 0$ and $H(\lambda, \gamma) \succ 0$. With $X \mapsto \sigma(X):=(1, X)^{T} H(\lambda, \gamma)\left[\begin{array}{c}1 \\ X\end{array}\right] \in \Sigma_{2}^{2}$, one obtains a strictly feasible solution $(\gamma, \lambda, \sigma)$ of $\mathbf{Q}^{*}$. As the value of both primal and dual strictly feasible solutions are finite, it follows that there is no duality gap, i.e., $\min \mathbf{Q}=\max \mathbf{Q}^{*}$, and both $\mathbf{Q}$ and $\mathbf{Q}^{*}$ are solvable.

Next, zero-duality gap yields complementarity ${ }^{2}$ at optimal solutions $y^{*}$ and $\left(\gamma^{*}, \lambda^{*}, \sigma^{*}\right)$ of $\mathbf{Q}$ and $\mathbf{Q}^{*}$, i.e., $\operatorname{trace}\left(M_{1}\left(y^{*}\right) H\left(\lambda^{*}, \gamma^{*}\right)\right)=0$. Therefore $H\left(\lambda^{*}, \gamma^{*}\right)$ must be singular. Notice that $H(\lambda, \gamma) \succeq 0$ implies that

$$
-2 A_{\lambda} u=\mathbf{f}
$$

for some $u \in \mathbb{R}^{n}$ and $\gamma+\lambda_{1} c_{1}+\lambda_{2} c_{2} \leq u^{T} A_{\lambda} u$.

We next prove that generically (i.e., except perhaps for a set of vectors $\{\mathbf{f}\} \subset \mathbb{R}^{n}$ with zero Lebesgue measure) $A_{\lambda^{*}} \prec 0$, and so $\operatorname{rank} H\left(\lambda^{*}, \gamma^{*}\right)=$ $n-1$. Indeed, consider the set of $\lambda \in \mathbb{R}_{+}^{2}$ with $\lambda_{1} \lambda_{2} \neq 0$, such that $A_{\lambda}$ is singular. Equivalently, after scaling by $\rho:=\lambda_{1}+\lambda_{2}>0$, and letting $\alpha:=\lambda_{1} /\left(\lambda_{1}+\lambda_{2}\right)$, the set of $\alpha \in[0,1]$ such that the determinant of the real symmetric matrix $B:=A_{2}+\alpha\left(A_{1}-A_{2}\right)$ vanishes. Such an $\alpha$ must be a root in $[0,1]$ of the characteristic polynomial of $B$, which has at most $n$

\footnotetext{
${ }^{2}$ See for instance Alizadeh et al. [1] or Pataki and Tuncel [15]
} 
solutions $\left(\alpha_{k}\right)$. So $A_{\lambda}$ is singular only on the (at most $n$ ) rays $\left(\lambda_{1}^{k}, \lambda_{2}^{k}\right)=$ $\rho\left(\alpha_{k}, 1-\alpha_{k}\right)$, with $\rho \geq 0$ and $\alpha_{k} \in[0,1]$. For each $\alpha_{k}$, the image space of $A_{\lambda^{k}}=\rho\left(\alpha_{k} A_{1}+\left(1-\alpha_{k}\right) A_{2}\right)$ is at most $(n-1)$-dimensional, and (2.21) holds if and only if

$$
\mathbf{f}=-2 \rho\left(\alpha_{k} A_{1}+\left(1-\alpha_{k}\right) A_{2}\right) u,
$$

for some $u \in \mathbb{R}^{n}$, i.e., if and only if

$$
v_{j}^{T} \mathbf{f}=0, \quad j=1, \ldots, p
$$

where $\left(v_{j}\right)_{j=1}^{p}$ is a basis of $\operatorname{Ker}\left(\alpha_{k} A_{1}+\left(1-\alpha_{k}\right) A_{2}\right)$.

If $p \geq 1$ then there is no solution in general, except perhaps on a set $\{\mathbf{f}\}_{k} \subset \mathbb{R}^{n}$ of zero Lebesgue measure. Therefore, as the set $\cup_{k}\{\mathbf{f}\}_{k}$ has zero Lebesgue measure, $A_{\lambda^{*}} \prec 0$ at an optimal solution $\lambda^{*}>0$, for generic $\mathbf{f} \in \mathbb{R}^{n}$. Similar arguments are also valid if $\lambda_{1}=0$ or $\lambda_{2}=0$, as $\mathbf{f}$ must belong to the image space of $A_{1}$ or $A_{2}$.

And so, $H\left(\lambda^{*}, \gamma^{*}\right)$ has only one zero eigenvalue, which by complementarity, implies that $M_{1}\left(y^{*}\right)$ is rank-one. This in turn implies the desired result $\min \mathbf{Q}=f^{*}$.

(b) From $\min \mathbf{Q}=\max \mathbf{Q}^{*}=f^{*}$, for generic $\mathbf{f} \in \mathbb{R}^{n}$ and $\mathbf{c} \in \mathbb{R}^{2}$

$$
f-f^{*}=\sigma^{*}+\lambda_{1}^{*} g_{1}+\lambda_{2}^{*} g_{2},
$$

for some $\lambda^{*} \in \mathbb{R}_{+}^{2}$ and some $\sigma^{*} \in \Sigma^{2}$ of degree 2 , that is, $f-f^{*} \in Q_{1}(g)$. In other words, the PP-BDR property holds for $\mathbf{K}$ with order $r=1$, and so, $\operatorname{co}(\mathbf{K})$ has the SDr (2.12) which is the same as that of Theorem 3(b).

Figures 1, 2 and 3 respectively, display three examples of sets $\mathbf{K}_{1}, \mathbf{K}_{2}, \mathbf{K}_{3} \subset$ $\mathbb{R}^{2}$ that have the PP-BDR property with order $r=1$. In all cases $g_{1}(X)=$ $1-X_{1}^{2}-X_{2}^{2}$, and

$$
\begin{aligned}
g_{2}(X) & =\left(X_{1}-1\right)^{2}+X_{2}^{2}-1 & & {\left[\text { for } \mathbf{K}_{1}\right] } \\
& =1 / 8-X_{1} X_{2} & & {\left[\text { for } \mathbf{K}_{2}\right] } \\
& =X_{1} X_{2}-1 / 8 & & {\left[\text { for } \mathbf{K}_{3}\right] . }
\end{aligned}
$$

Notice that $\mathbf{K}_{3}$ is not even connected, and that for $\mathbf{K}_{1}$, one even has a linear term $X_{1}$ in the polynomial $g_{2}$.

Remark 4. Theorem 3 illustrates the fact that the PP-BDR property is specific to the representation of affine polynomials. Indeed if $f \in \mathbb{R}[X]$ is now an arbitrary quadratic polynomial $X \mapsto f(X)=X^{T} A_{0} X+\mathbf{f}^{T} x+f_{0}$, then in general (and except in some special cases as those treated in [21]) $f-f^{*} \notin Q_{1}(g)$ even for generic data $A_{0}$, f. See for instance some complexity results in quadratic optimization in Ye and Zhang [21].

Example 5. With $\mathbf{K}$ as in (1.3), let $\widehat{\mathbf{K}}:=\mathbf{K} \cap\{-1,1\}^{n}$. The results in Lasserre $[9,10]$ show that $\widehat{\mathbf{K}}$ has the PP-BDR property with order $r=$ $n+\max _{j}\left\lceil\operatorname{deg} g_{j} / 2\right\rceil$. Hence $\operatorname{co}(\widehat{\mathbf{K}})$ has the $\mathrm{SDr}(2.12)$ with the additional constraints $y_{\alpha}=y_{\alpha \bmod 2}$ for all $\alpha$. In this case, the PP-BDR property is not useful for practical purposes because $r$ depends on $n$, and the corresponding SDP has $2^{n}$ variables $y_{\alpha}$. 


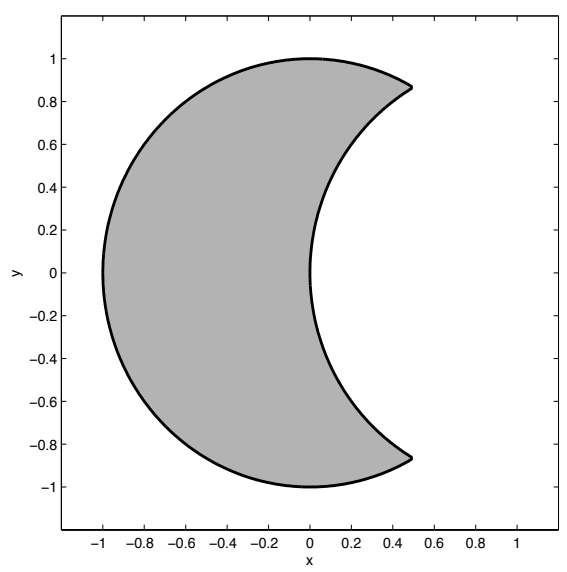

Figure 1. $\mathbf{K}_{1}: g_{2}(X)=\left(X_{1}-1\right)^{2}+X_{2}-1$

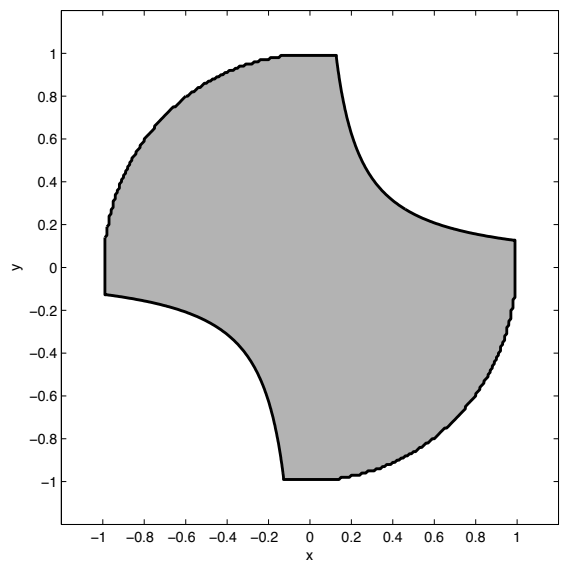

Figure 2. $\mathbf{K}_{2}: g_{2}(X)=1 / 8-X_{1} X_{2}$

2.5. An approximate $\mathbf{S D r}$ set. With $\mathbf{B}:=\left\{x \in \mathbb{R}^{n}:\|x\| \leq 1\right\}$ and given a compact set $\Omega \subset \mathbb{R}^{n}$ and $\rho>0$, let

$$
\Omega+\rho \mathbf{B}=\left\{x \in \mathbb{R}^{n} \mid \inf _{y \in \Omega}\|x-y\| \leq \rho\right\} .
$$

In this section we prove that given any $\epsilon>0$, there is a convex SDr set in sandwich between $\operatorname{co}(\mathbf{K})$ and $\operatorname{co}(\mathbf{K})+\epsilon \mathbf{B}$ and with an explicit SDr in terms of the $g_{j}$ 's that define $\mathbf{K}$. For this purpose we use a result of Prestel (later refined by Schweighofer [18]) on a degree bound in Schmüdgen's Positivstellensatz (and similarly a result of Nie and Schweighofer [19] on a degree bound in Putinar's Positivstellensatz).

We first need the following intermediate result.

Lemma 5. (a) Let $\Omega \subset \mathbb{R}^{n}$ be a compact convex set and let $\epsilon>0$ be fixed. If $x \notin \Omega+\epsilon \mathbf{B}$ then there exists a linear $f \in \mathbb{R}[X]$ whose coefficient vector 


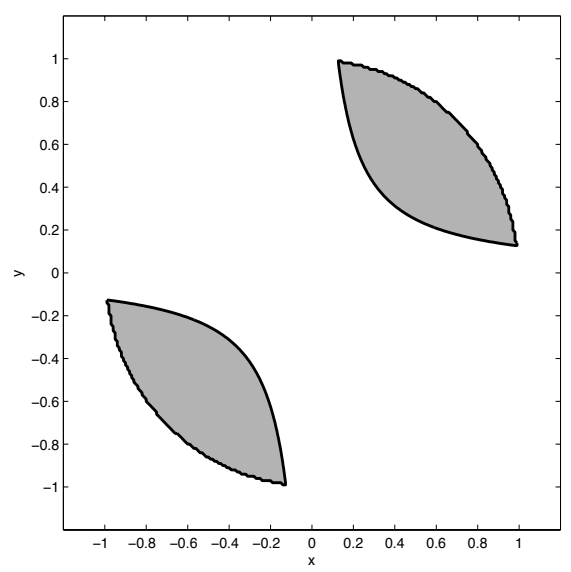

Figure $3 . \mathbf{K}_{2}: g_{2}(X)=X_{1} X_{2}-1 / 8$

$\mathbf{f} \in \mathbb{R}^{n}$ satisfies $\|\mathbf{f}\|=1$, and a scalar $f^{*}$ such that

$$
f(z) \geq f^{*} \quad \forall z \in \Omega \quad \text { and } \quad f(x)<f^{*}-\epsilon .
$$

In addition, $\left|f^{*}\right| \leq \tau_{\Omega}:=\max \{\|x\|: x \in \Omega\}$.

(b) For any compact set $\mathbf{K} \subset \mathbb{R}^{n}$, and any $\mathbf{f} \in \mathbb{R}^{n}$ with $\|\mathbf{f}\|=1$, let $f^{*}:=\min _{x \in \mathbf{K}} \mathbf{f}^{T} x$, and let $\tau_{\mathbf{K}}:=\max \{\|x\|: x \in \mathbf{K}\}$. Then

$$
\min _{x \in \operatorname{co}(\mathbf{K})} \mathbf{f}^{T} x=f^{*} \quad \text { and } \quad\left|f^{*}\right| \leq \tau_{\mathbf{K}} .
$$

Proof. (a) With $x \notin \Omega+\epsilon \mathbf{B}$, let $x^{*} \in \Omega$ be its projection on $\Omega$ (well defined because $\Omega$ is compact and convex). Let $\mathbf{f}:=\left(x^{*}-x\right) /\left\|x-x^{*}\right\|$ so that $\|\mathbf{f}\|=1$, and let $f^{*}:=\mathbf{f}^{T} x^{*}$, so that $\left|f^{*}\right| \leq\|\mathbf{f}\| \max \{\|x\|: x \in \Omega\}=\tau_{\Omega}$.

Then with $f \in \mathbb{R}[X]$ being the linear polynomial with coefficient vector f, one has $f(z) \geq f^{*}$ for all $z \in \Omega$ because

$$
f(z)=\mathbf{f}^{T} z=\mathbf{f}^{T} x^{*}+\mathbf{f}^{T}\left(z-x^{*}\right)=f^{*}+\left\langle x \vec{x}^{*}, x^{*} z\right\rangle \geq f^{*}
$$

(since $\left\langle x \vec{x}^{*}, x^{*} z\right\rangle \geq 0$ ), and

$$
f(x)-f^{*}=\mathbf{f}^{T}\left(x-x^{*}\right)=-\left\|x-x^{*}\right\|<-\epsilon .
$$

(b) Indeed, $f^{*}=\min _{x \in \mathbf{K}} \mathbf{f}^{T} x=\min _{x \in \operatorname{co}(\mathbf{K})} \mathbf{f}^{T} x$. Moreover, $\left|\mathbf{f}^{T} x\right| \leq\|\mathbf{f}\| \cdot\|x\| \leq \tau_{\mathbf{K}}$ for all $x \in \mathbf{K}$.

Then we have the following result.

Theorem 6. Let $\mathbf{K} \subset \mathbb{R}^{n}$ be a compact set as defined in (1.3). 
(a) For every fixed $\epsilon>0$ there is a integer $r_{\epsilon} \in \mathbb{N}$ such that the SDr set $\mathbf{K}_{\epsilon}$ defined by

$$
\mathbf{K}_{\epsilon}:=\left\{x \in \mathbb{R}^{n}:\left\{\begin{array}{lll}
\exists y \in \mathbb{R}^{s\left(2 r_{\epsilon}\right)}: & & \\
M_{r_{\epsilon}-r_{J}}\left(g_{J} y\right) & \succeq & 0, \quad J \subseteq\{1, \ldots, m\} \\
L_{y}\left(X_{i}\right) & =x_{i}, & i=1, \ldots, n \\
y_{0} & =1
\end{array}\right\}\right.
$$

satisfies $\operatorname{co}(\mathbf{K}) \subseteq \mathbf{K}_{\epsilon} \subset \mathbf{K}+\epsilon \mathbf{B}$.

(b) Assume that the polynomial $N-\|X\|^{2}$ is in the quadratic module $Q(g)$. Then for every fixed $\epsilon>0$ there is an integer $r_{\epsilon} \in \mathbb{N}$ such that the SDr set $\mathbf{K}_{\epsilon}$ defined by

$$
\mathbf{K}_{\epsilon}:=\left\{x \in \mathbb{R}^{n}:\left\{\begin{array}{ll}
\exists y \in \mathbb{R}^{s\left(2 r_{\epsilon}\right)}: & \\
M_{r_{\epsilon}-r_{j}}\left(g_{j} y\right) & \succeq 0, \quad j=0, \ldots, m \\
L_{y}\left(X_{i}\right) & =x_{i}, \quad i=1, \ldots, n \\
y_{0} & =1
\end{array}\right\}\right.
$$

satisfies $\operatorname{co}(\mathbf{K}) \subseteq \mathbf{K}_{\epsilon} \subset \mathbf{K}+\epsilon \mathbf{B}$.

In both cases (a) and (b), bounds on $r_{\epsilon}$ are available.

Proof. (a) That $\operatorname{co}(\mathbf{K}) \subseteq \mathbf{K}_{\epsilon}$ is straightforward and as in the proof of Theorem 2. Next, let $x \notin \operatorname{co}(\mathbf{K})+\epsilon \mathbf{B}$ be fixed. Then by Lemma 5 (with $\Omega:=\operatorname{co}(\mathbf{K}))$ there exists $\mathbf{f} \in \mathbb{R}^{n}$ and $f^{*}:=\min _{x \in \operatorname{co}(\mathbf{K})} \mathbf{f}^{T} x$ such that (2.23) holds. In addition, $\|\mathbf{f}\|=1$ and $\left|f^{*}\right| \leq \tau_{\mathbf{K}}$.

Let $f \in \mathbb{R}[X]$ be the affine polynomial with coefficient vector $\left(\mathbf{f},-f^{*}\right) \in$ $\mathbb{R}^{n} \times \mathbb{R}$ so that $f+\epsilon \geq \epsilon>0$ on $\mathbf{K}$. By Schmüdgen Positivstellensatz [17], $f+\epsilon \in P(g)$. Even more, $f+\epsilon \in P_{r_{\epsilon}}(g)$ for some integer $r_{\epsilon} \in \mathbb{N}$ that does not depend on the precise value of $f$ but only on its degree (here 1) and norm (here $\|\mathbf{f}\|=1$ and $\left|f^{*}\right| \leq \tau_{\mathbf{K}}$ ); see Schweighofer [18]. So let $\mathbf{K}_{\epsilon}$ be the SDr set defined in (2.25) with this $r_{\epsilon}$. If $x \in \mathbf{K}_{\epsilon}$, we obtain the contradiction

$$
\begin{aligned}
0> & \mathbf{f}^{T} x-f^{*}+\epsilon \\
& =\left(\epsilon-f^{*}\right) y_{0}+\sum_{i=1}^{n} L_{y}\left(f_{i} X_{i}\right) \quad\left[\text { as } y_{0}=1 \text { and } L_{y}\left(X_{i}\right)=x_{i} \quad \forall i\right] \\
& =L_{y}(f(X)+\epsilon)=\sum_{J \subseteq\{1, \ldots, m\}} L_{y}\left(g_{J} \sigma_{J}\right) \quad\left[\text { as } f+\epsilon \in P_{r_{\epsilon}}(g)\right] \\
& \geq 0 \quad[\text { by }(2.15)] .
\end{aligned}
$$

Hence $\mathbf{K}_{\epsilon} \subset \operatorname{co}(\mathbf{K})+\epsilon \mathbf{B}$, the desired result.

(b) The proof is very similar except that now we invoke Putinar Positivstellenstaz [16] and Nie and Schweighofer [19] to replace $P_{r_{\epsilon}}(g)$ with $Q_{r_{\epsilon}}(g)$. Finally, bounds on $r_{\epsilon}$ can be found for both cases (a) and (b) in [18] and [19] respectively.

Hence, no matter if $\operatorname{co}(\mathbf{K})$ is SDr, for every $\epsilon>0$, there is always a SDr set $\mathbf{K}_{\epsilon}$ in sandwich between $\operatorname{co}(\mathbf{K})$ and $\operatorname{co}(\mathbf{K})+\epsilon \mathbf{B}$. In addition, the SDr of $\mathbf{K}_{\epsilon}$ is explicit in terms of the polynomials $\left(g_{j}\right)$ that define $\mathbf{K}$. This is a 
significant improvement upon the outer convex approximations $\Delta_{r} \downarrow \operatorname{co}(\mathbf{K})$ of [8], where each $\Delta_{r}$ has a SDr. Indeed in [8], if $x \notin \operatorname{co}(\mathbf{K})$ then $x \notin \Delta_{r}$ for all $r \geq r(x)$ for some $r(x)$ that depends on $x$, an undesirable feature.

\section{II. SDR FOR COMPACT CONVEX BASIC SEMIALGEBRAIC SETS}

In this section, $\mathbf{K} \subset \mathbb{R}^{n}$ defined in (1.3) is compact and convex, and we assume that one knows a scalar $\tau_{\mathbf{K}}$ such that:

$$
x \in \mathbf{K} \quad \Rightarrow \quad\|x\| \leq \tau_{\mathbf{K}} .
$$

Lemma 7. Let $\mathbf{K} \subset \mathbb{R}^{n}$ be as in (1.3), and assume that the $g_{j}$ 's that define $\mathbf{K}$ are all concave and Slater's condition holds. Given $f \in \mathbb{R}[X]$, let $f^{*}:=\min _{\in \mathbf{K}} f(x)$.

For every linear $f \in \mathbb{R}[X]$ with $\|\mathbf{f}\|=1$, there exists $\lambda(f) \in \mathbb{R}_{+}^{m}$ such that

$$
\begin{aligned}
X \mapsto L_{f}(X) & :=f(X)-f^{*}-\sum_{j=1}^{m} \lambda_{j}(f) g_{j}(X) \geq 0 \quad \text { on } \mathbb{R}^{n} \\
\left|f^{*}\right| & \leq \tau_{\mathbf{K}} ; \quad \lambda_{j}(f) \leq M_{\mathbf{K}}, \quad j=1, \ldots, m,
\end{aligned}
$$

where $M_{\mathbf{K}}$ is independent of $f$.

Proof. As the $g_{j}$ 's are concave, $\mathbf{K}$ is compact and convex. In addition, as Slater's condition holds and $f$ is convex, there exist nonnegative Lagrange multipliers $\lambda(f) \in \mathbb{R}_{+}^{m}$ such that

$$
\nabla f\left(x^{*}\right)=\sum_{j=1}^{m} \lambda_{j}(f) \nabla g_{j}\left(x^{*}\right) ; \quad \lambda_{j}(f) g_{j}\left(x^{*}\right)=0, \forall j=1, \ldots, m,
$$

where $x^{*} \in \mathbf{K}$ is a (global) minimizer of $f$ on $\mathbf{K}$. Therefore the Lagrangian $L_{f}$ defined in (3.2) is convex, with $f^{*}$ as its global minimum on $\mathbb{R}^{n}$ and $x^{*}$ as global minimizer. Recall that Slater's condition states that $g_{j}\left(x_{0}\right)>0$, $j=1, \ldots, m$, for some $x_{0}$. And so, from

$$
L_{f}\left(x_{0}\right)=f\left(x_{0}\right)-f^{*}-\sum_{j=1}^{m} \lambda_{j}(f) g_{j}\left(x_{0}\right) \geq 0,
$$

we deduce that for every $j=1, \ldots, m$,

$$
0 \leq \lambda_{j}(f) \leq \frac{f\left(x_{0}\right)-f^{*}}{g_{j}\left(x_{0}\right)} \leq \frac{2 \tau_{\mathbf{K}}}{g_{j}\left(x_{0}\right)} \leq \frac{2 \tau_{\mathbf{K}}}{\min _{j=1, \ldots, m} g_{j}\left(x_{0}\right)}=: M_{\mathbf{K}},
$$

where we have used $\|\mathbf{f}\|=1$. Therefore (3.3) holds and $M_{\mathbf{K}}$ above is independent of $f$.

Theorem 8. Let $\mathbf{K} \subset \mathbb{R}^{n}$ be compact and defined as in (1.3). Assume that the $g_{j}$ 's that define $\mathbf{K}$ are all concave and Slater's condition holds. Given a linear polynomial $f \in \mathbb{R}[X]$, let $L_{f}$ be the Lagrangian defined in (3.2). 
If $L_{f}$ is s.o.s. for every linear $f \in \mathbb{R}[X]$, then the PP-BDR property holds for $\mathbf{K}$ with order $r=\max _{j=1, \ldots, m}\left\lceil\operatorname{deg} g_{j} / 2\right\rceil$, and $\mathbf{K}$ is a $S D r$ set. In addition, the convex set

$$
\boldsymbol{\Omega}:=\left\{(x, y) \in \mathbb{R}^{n} \times \mathbb{R}^{s(2 r)}:\left[\begin{array}{lll}
M_{r}(y) & \succeq 0 & \\
L_{y}\left(g_{j}\right) & \geq 0, & j=1, \ldots, m \\
L_{y}\left(X_{i}\right) & =x_{i}, & i=1, \ldots, n \\
y_{0} & =1
\end{array}\right\}\right.
$$

is a $S D r$ of $\mathbf{K}$.

Proof. Let $x \in \mathbf{K}$ and let $y=\left(x^{\alpha}\right) \in \mathbb{R}^{s(r)}$. Then $M_{r}(y) \succeq 0$ and $L_{y}\left(g_{j}\right)=$ $g_{j}(x) \geq 0$ for all $j=1, \ldots, m$. Therefore, $(x, y) \in \boldsymbol{\Omega}$.

Conversely, let $x \notin \mathbf{K}$, and suppose that $(x, y) \in \mathbf{\Omega}$ for some $y \in \mathbb{R}^{s(r)}$. As $x \notin \mathbf{K}$ there exists $\left(f^{*}, \mathbf{f}\right) \in \mathbb{R} \times \mathbb{R}^{n}$ with $\|\mathbf{f}\|=1$ such that $\mathbf{f}^{T} z \geq f^{*}$ for all $z \in \mathbf{K}$ and $\mathbf{f}^{T} x<f^{*}$. Actually, $f^{*}=\min _{x \in \mathbf{K}} f(x)$ where $f \in \mathbb{R}[X]$ is linear with vector of coefficients $\mathbf{f}$. Let $L_{f}$ be as in (3.2). If $L_{f}$ is s.o.s. then $f-f^{*}=\sigma+\sum_{j=1}^{m} \lambda_{j}(f) g_{j}$ for some s.o.s. polynomial $\sigma \in \mathbb{R}[X]$ of degree at most $2 r$. Therefore, one obtains the contradiction

$$
\begin{aligned}
0 & >\mathbf{f}^{T} x-f^{*}=f(x)-f^{*} \\
& \geq L_{y}\left(f-f^{*}\right)=L_{y}\left(\sigma+\sum_{j=1}^{m} \lambda_{j} g_{j}\right) \\
& \geq 0 \quad[\text { as }(x, y) \in \boldsymbol{\Omega}] .
\end{aligned}
$$

Remark 9. Interestingly, Theorem 8 has a rephrasing in terms of the support function $\mathbf{f} \mapsto \sigma_{\mathbf{K}}(\mathbf{f})$ of $\mathbf{K}$, defined by:

$$
\mathbf{f} \mapsto \sigma_{\mathbf{K}}(\mathbf{f}):=\sup \{\langle\mathbf{f}, x\rangle: \quad x \in \mathbf{K}\} .
$$

For more details on the support function and its properties, the interested reader is referred to e.g. Hiriart-Urruty and Lemarechal [6, Chapter V].

For every linear polynomial $f \in \mathbb{R}[X]$, let $\mathbf{f} \in \mathbb{R}^{n}$ be its vector of coefficients. Then observe that in Theorem 8 , and with $r=\max _{j=1, \ldots, m}\left\lceil\operatorname{deg} g_{j} / 2\right\rceil$, the statement " $L_{f}$ is s.o.s. for every linear $f \in \mathbb{R}[X]$ " can be replaced with the new statement $" f+\sigma_{\mathbf{K}}(-\mathbf{f}) \in Q_{r}(g)$ for every linear $f \in \mathbb{R}[X]$ ".

The SDr (3.4) of $\mathbf{K}$ is very natural as it is based on the Karush-KuhnTucker optimality conditions. Existence of such a SDr reduces to the real algebraic problem of checking whether the Lagrangian $L_{f}$ is s.o.s. for every (in fact, almost all) linear $f \in \mathbb{R}[X]$. Examples 2 and 3 in $\S 2.3$ provide such instances of sets $\mathbf{K}$ with the PP-BDR property and with $\mathrm{SDr}$ (3.4).

Hence, an important issue to find sufficient conditions to ensure that the Lagrangian $L_{f}$ is s.o.s., and if possible, conditions that can be checked 
directly from the data $g_{j}$. For instance, in Lasserre [12] one finds sets of sufficient conditions on the coefficients of a polynomial $f$ to ensure it is s.o.s. Also, after the present paper was written, Helton and Nie [5] have provided several sufficient conditions for the Lagrangian $L_{f}$ to be s.o.s. In particular, if the Hessian $-\nabla^{2} g_{j}(X)$ can be written $P_{j}(X) P_{j}(X)^{T}$ for some (not necessarily square) matrix $P_{j}(X)$ (i.e. $-\nabla^{2} g_{j}(X)$ is a sum of squares), $j=1, \ldots, m$, then $L_{f}$ is s.o.s.

Example 6. Consider the class of convex sets $\mathbf{K} \subset \mathbb{R}^{n}$ with $g_{j} \in \mathbb{R}[X]$ concave and of the form

$$
g_{j}(X)=-\sum_{i=1}^{n} g_{j i} X_{i}^{2 d}+h_{j}(X)+g_{j 0}, \quad j=1, \ldots, m,
$$

with $\left(g_{j i}\right) \subset \mathbb{R}_{+}$, and $h_{j} \in \mathbb{R}[X]$ linear for every $j=1, \ldots, m$. Then $-\nabla^{2} g_{j}(X)$ is the diagonal matrix with diagonal elements $\left(g_{i i} X_{i}^{2 d-2}\right)$ and so can be written as $P_{j}(X) P_{j}(X)^{T}$ with $P_{j}(X)=\left(\nabla^{2} g_{j}(X)\right)^{1 / 2}$. Therefore, by Theorem 8, $\mathbf{K}$ has the $\operatorname{SDr}(3.4)$.

Taking $\mathbf{K}:=\left\{x \in \mathbb{R}^{n}:\|x\|_{d}\left(:=\left(\sum_{i=1}^{n} x_{i}^{2 d}\right)^{1 / 2 d}\right) \leq 1\right\}$ as a particular case of Example 6, one may thus conclude that the $p$-Euclidean ball is SDr, for all $d \geq 1$.

Approximate SDr. When one does not know whether the Lagrangian $L_{f}$ is s.o.s. for all (in fact, almost all) linear $f \in \mathbb{R}[X]$, we next provide an approximation result. Namely we provide a semidefinite representation $\boldsymbol{\Omega}_{\mathbf{r}}$ for an arbitrarily close convex approximation $\mathbf{K}_{r}$ of $\mathbf{K}$. This approximation is in the spirit of that of $\S 2.5$, but specific to the convex case. We first need the following crucial auxiliary results.

Lemma 10. Let $\mathbf{K} \subset \mathbb{R}^{n}$ be as in (1.3), $\tau_{\mathbf{K}}$ as in (3.1), and assume that the $g_{j}$ 's that define $\mathbf{K}$ are all concave and Slater's condition holds. Let $X \mapsto \Theta_{r}(X):=\sum_{i=1}^{n}\left(\frac{X_{i}}{\tau_{\mathrm{K}}}\right)^{2 r}$.

Then for every $\epsilon>0$ there exists $r(\epsilon)$ such that for every linear $f \in \mathbb{R}[X]$ with $\|\mathbf{f}\|=1$ and $L_{f}$ as in (3.2),

$$
L_{f}+\epsilon\left(1+\Theta_{r}\right) \quad \text { is s.o.s. } \forall r \geq r(\epsilon) .
$$

Equivalently, $f-f^{*}+\epsilon\left(1+\Theta_{r}\right) \in Q_{r}(g)$.

Proof. By Lemma $7, L_{f} \geq 0$ and observe that the coefficients of the polynomial $L_{f}$ are all uniformly bounded in $\mathbf{f}$ whenever $\|\mathbf{f}\|=1$. Indeed,

$$
0 \leq \lambda_{j}(f) \leq M_{\mathbf{K}} \quad \forall j=1, \ldots, m ; \quad\left|f^{*}\right| \leq \tau_{\mathbf{K}},
$$

with $\tau_{\mathbf{K}}$ as in (3.1) and $M_{\mathbf{K}}$ as in Lemma 7 . Hence, in view of the definition (3.2) of the polynomial $L_{f}$, its coefficients $\left(L_{f}\right)_{\alpha}$ are all bounded, uniformly in $\mathbf{f}$.

Next, $L_{f} \geq 0$ implies that $L_{f}$ is nonnegative on the box $\left[-\tau_{\mathbf{K}}, \tau_{\mathbf{K}}\right]^{n}$. Therefore (3.6) follows from Lasserre and Netzer [11, §3.3], where it was 
proved that the degree $r(\epsilon)$ does not depend on the precise value of the coefficients of $L_{f}$ but only on $\epsilon$, the dimension $n$, the degree of $L_{f}$ and the size of its coefficients. Here, whenever $\mathbf{f}$ varies, the degree of $L_{f}$ takes finitely many values (depending on which Lagrange multipliers $\lambda_{j}$ are zero), and its coefficients are uniformly bounded.

Next, in view of (3.1) and with $\Theta_{r} \in \mathbb{R}[X]$ as in Lemma 10,

$$
\Theta_{r}(x) \leq 1 \quad \forall x \in \mathbf{K}, \quad \forall r \in \mathbb{N} .
$$

Theorem 11. Let $\mathbf{K} \subset \mathbb{R}^{n}$ as in (1.3) be compact, with $\tau_{\mathbf{K}}$ as in (3.1). Assume that the $g_{j}$ 's that define $\mathbf{K}$ are all concave and Slater's condition holds. With $r \in \mathbb{N}, r \geq\left\lceil\operatorname{deg} g_{j} / 2\right\rceil, j=1, \ldots, m$, let $\Theta_{r}(X)=\sum_{i=1}^{n}\left(\frac{X_{i}}{\tau_{\mathrm{K}}}\right)^{2 r}$, and let $\mathbf{K}_{r} \subset \mathbb{R}^{n}$ be the convex set:

$$
\mathbf{K}_{r}:=\left\{x \in \mathbb{R}^{n}:\left[\begin{array}{ll}
\exists y \in \mathbb{R}^{s(2 r)} & \text { s.t. } \\
M_{r}(y) & \succeq 0 \\
L_{y}\left(g_{j}\right) & \geq 0, \quad j=1, \ldots, m \\
L_{y}\left(\Theta_{r}\right) & \leq 1 \\
L_{y}\left(X_{i}\right) & =x_{i}, \quad i=1, \ldots, n \\
y_{0} & =1
\end{array}\right\} .\right.
$$

Then for every $\epsilon>0$, there exists $r \in \mathbb{N}$ such that

$$
\mathbf{K} \subseteq \mathbf{K}_{\mathbf{r}} \subseteq \mathbf{K}+\epsilon \mathbf{B},
$$

and the convex set

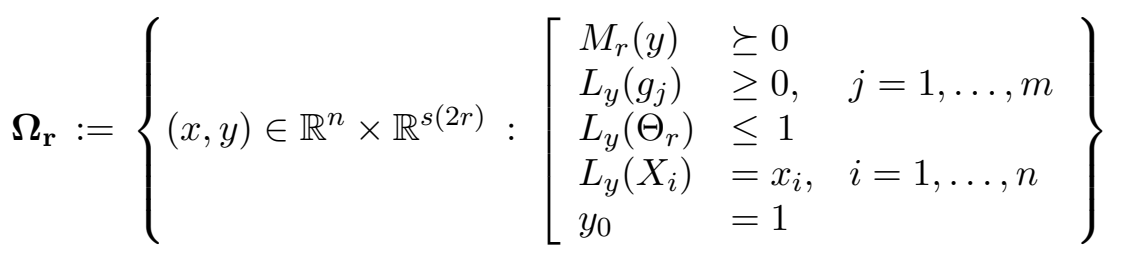

is a SDr of $\mathbf{K}_{r}$.

Proof. Let $x \in \mathbf{K}$. Then the vector $y=\left(x^{\alpha}\right) \in \mathbb{R}^{s(r)}$ satisfies the constraints described in (3.8), so that $\mathbf{K} \subseteq \mathbf{K}_{r}$ for all $r \geq\left\lceil\operatorname{deg} g_{j} / 2\right\rceil, j=1, \ldots, m$.

To prove $\mathbf{K}_{r} \subseteq \mathbf{K}+\epsilon \mathbf{B}$, we proceed by contradiction. With $\epsilon>0$ fixed, let $x \notin \mathbf{K}+\epsilon \mathbf{B}$ be fixed but arbitrary, and with $r(\epsilon / 2)$ as in Lemma 10, let $r \geq r(\epsilon / 2)$ be fixed arbitrary. Let $f \in \mathbb{R}[X]$ be as in Lemma 5 so that $f(x)-f^{*}<-\epsilon$. Next, with $L_{f}$ being the Lagrangian associated with $f$, by Lemma 10,

$$
L_{f}+\frac{\epsilon}{2}\left(1+\Theta_{r}\right)=\sigma,
$$

for some s.o.s. polynomial $\sigma \in \mathbb{R}[X]$ of degree $2 r$. Equivalently,

$$
f-f^{*}+\frac{\epsilon}{2}\left(1+\Theta_{r}\right)=\sigma+\sum_{j=1}^{m} \lambda_{j}(f) g_{j} .
$$


Now, suppose that $x \in \mathbf{K}_{r}$. There exists $y \in \mathbb{R}^{s(r)}$ such that $(x, y) \in \mathbf{\Omega}_{\mathbf{r}}$. In particular, $L_{y}\left(\Theta_{r}\right) \leq 1, L_{y}\left(g_{j}\right) \geq 0, j=1, \ldots, m$, and $L_{y}(\sigma) \geq 0$ for every $\sigma \in \Sigma_{r}^{2}$ (because $M_{r}(y) \succeq 0$ ). And so, we obtain the contradiction

$$
\begin{aligned}
0>\mathbf{f}^{T} x-f^{*}+\epsilon & =f(x)-f^{*}+\frac{\epsilon}{2}+\frac{\epsilon}{2} \\
& \geq L_{y}\left(f-f^{*}\right)+\frac{\epsilon}{2} L_{y}\left(1+\Theta_{r}\right) \\
& =L_{y}(\sigma)+\sum_{j=1}^{m} \lambda_{j}(f) L_{y}\left(g_{j}\right) \quad[\text { by }(3.11)] \\
& \geq 0 \quad\left[\text { as }(x, y) \in \mathbf{\Omega}_{\mathbf{r}}\right] .
\end{aligned}
$$

Therefore $x \notin \mathbf{K}_{r}$. As $x \notin \mathbf{K}+\epsilon \mathbf{B}$ was arbitrary, this implies $\mathbf{K}_{r} \subseteq \mathbf{K}+\epsilon \mathbf{B}$. Finally, that $\boldsymbol{\Omega}_{\mathbf{r}}$ in (3.10) is a SDr of $\mathbf{K}_{r}$, follows from the definition (3.8) of $\mathbf{K}_{r}$.

The SDr $\boldsymbol{\Omega}_{\mathrm{r}}$ of the convex set $\mathbf{K}_{r}$ in Theorem 11 resembles the SDr $\boldsymbol{\Omega}$ of $\mathbf{K}$ in Theorem 8. The only difference is the index $r$ which is larger than $\max _{j}\left\lceil\operatorname{deg} g_{j} / 2\right\rceil$, and the additional constraint $L_{y}\left(\Theta_{r}\right) \leq 1$.

Hence, it is worth noticing that if $\mathbf{K}$ does not admit the SDr $\boldsymbol{\Omega}$ of Theorem 8 , one still obtains a SDr $\boldsymbol{\Omega}_{\mathbf{r}}$ of an arbitrarily close convex approximation $\mathbf{K}_{r}$ of $\mathbf{K}$, explicit in terms of the concave polynomials $\left(g_{j}\right)$ that define $\mathbf{K}$.

\section{Conclusion}

We have considered the class of compact basic semialgebraic sets $\mathbf{K} \subset \mathbb{R}^{n}$, and have provided sufficient conditions for its convex hull $\operatorname{co}(\mathbf{K})$ to have a SDr expressed directly in terms of the polynomials that define $\mathbf{K}$. When $\mathbf{K}$ is convex and defined by concave polynomials, we have shown that if for every linear polynomial $f \in \mathbb{R}[X]$, the associated (nonnegative) Lagrangian $L_{f}$ is s.o.s., then $\mathbf{K}$ has a simpler specific SDr. Finally, we have also provided a SDr of an arbitrarily close approximation $\mathbf{K}_{\epsilon}$ of $\operatorname{co}(\mathbf{K})$ (and of $\mathbf{K}$ in the convex case). An interesting issue of further investigation is to provide concrete conditions on the concave polynomials $g_{j}$ 's, to ensure that the Lagrangian $L_{f}$ is s.o.s. The work in [5] provides some interesting results in this direction.

Acknowledgement: This work was supported by french ANR-grant NT05$3-41612$.

\section{REFERENCES}

[1] F. Alizadeh, J-P. Haeberly, M. Overton. Complementarity and nondegeneracy in semidefinite programming, Math. Programming 77 (1997), 111-128.

[2] A. Ben-Tal, A. Nemirovski. Lectures on Modern Convex Optimization, SIAM, Philadelphia, 2001.

[3] C. Beng Chua, L. Tuncel. Invariance and efficiency of convex representations, Math. Programming 111 (2008), 113-140. 
[4] J.W. Helton, V. Vinnikov. Linear matrix inequality representation of sets, Comm. Pure Appl. Math., to appear. arXiv:math.0C/0306180.

[5] J.W. Helton, J. Nie. Semidefinite representation of convex sets, Technical report, Mathematics Dept., University of California at San Diego, USA, 2007. arXiv:0705.4068.

[6] J-B. Hiriart-Urruty, C. Lemarechal. Convex Analysis and Minimization Algorithms I, Springer-Verlag, Berlin, 1993.

[7] T. Jacobi, A. Prestel. Distinguished representations of strictly positive polynomials, J. Reine. Angew. Math. 532 (2001), 223-235.

[8] R. Laraki, J.B. Lasserre. Computing uniform convex approximations for convex envelopes and convex hulls, J. of Convex Analysis, to appear.

[9] J.B. Lasserre. Global optimization with polynomials and the problem of moments, SIAM J. Optim. 11 (2001), 796-817.

[10] J.B. Lasserre. An explicit equivalent positive semidefinite program for nonlinear 0-1 programs, SIAM J. Optim. 12 (2002), 756-769.

[11] J.B. Lasserre, T. Netzer. SOS approximation of nonnegative polynomial via simple high degree perturbations, Math. Z. 256 (2006), 99-112.

[12] J.B. Lasserre. Conditions for a real polynomial to be sum of squares, Archiv der Mathematik, to appear. doi: 10.1007/s00013-007-2251-y Available at http://arxiv.org/abs/math. AG/0612358

[13] A.S. Lewis, P. Parrilo, M.V. Ramana. The Lax conjecture is true, Proc. Am. Math. Soc., 133 (2005), 2495-2499.

[14] P. Parrilo, Exact semidefinite representations for genus zero curves, Talk at the Banff workshop "Positive Polynomials and Optimization", Banff, Canada, October 8-12th 2006.

[15] G. Pataki, L. Tuncel. On the generic properties of convex optimization problems in conic form, Math. Programming 89 (2001), 449-457.

[16] M. Putinar. Positive polynomials on compact semi-algebraic sets, Indiana Univ. Math. J. 42 (1993), 969-984.

[17] K. Schmüdgen. The $\mathbb{K}$-moment problem for compact semi-algebraic sets, Math. Ann. 289 (1991), 203-206.

[18] M. Schweighofer. On the complexity of Schmdgen's Positivstellensatz, J. Complexity 20 (2004), pp. 529-543.

[19] Jiawang Nie, M. Schweighofer. On the complexity of Putinar's Positivstellensatz, J. Complexity 23 (2007), pp. 135-150.

[20] L. Vandenberghe, S. Boyd. Semidefinite programming, SIAM Review 38 (1996), pp. 49-95.

[21] Y. Ye, S. Zhang, New results on quadratic minimization, SIAM J. Optim. 14 (2003), $245-267$.

LAAS-CNRS and Institute of Mathematics, LAAS, 7 AVEnue du Colonel Roche, 31077 Toulouse Cedex 4, France

E-mail address: lasserre@laas.fr 